\title{
Serum Creatinine Level and Its Relation to Backache in Chronic Kidney Disease with Unknown Aetiology (CKDu) Patients in North Central Province, Sri Lanka
}

Authors: Fahim Aslam ${ }^{1}$ and Nishantha Kumarasinghe
fahimaslam30@gmail.com
A.F.MohamedAslam@wlv.ac.uk
drkumarasinghe2015@gmail.com

Faculty of Science and Engineering, University of Wolverhampton 


\begin{abstract}
$\underline{\text { Abstract }}$
Background - Chronic kidney disease with unknown aetiology $(\mathrm{CKDu})$ is a progressing disease which is affecting many Sri Lankans' yearly, this disease is slow progressive, irreversible and asymptomatic disease. Patients suffering from $\mathrm{CKDu}$ over the past two decades have been evaluated using the estimated glomerular filtration rate (e-GFR). This is the standard test performed under WHO guidelines to investigate and grade the CKDu, and this creatinine clearance is measured in this in order to grade the patient. Backache has been a common symptom presented in most of the chronic kidney disease patients (CKD), patients with $\mathrm{CKDu}$ are liable to get backache due to the renal failure that takes place (Jayathilake et al., 2013; Redmon et al., 2016).
\end{abstract}

Objective - To detect a correlation between serum creatinine values and level of backache presented in the patient.

Method -Using an interview based questionnaire to assess patients' body condition and the degree of backache is assessed, 59 patients were part of the study for a period of five months and had an average age of 60.3 years. The questionnaire answers are scaled into four main types of backache from the least to the most painful based on the pain scale adapted from Kapkia. Serum creatinine is measured using an automated analyzer which is measured using Jaffe's reaction. Using Pearson R correlation, the relationship was determined between serum creatinine and backache (Kafkia et al., 2011).

Results - The results were obtained for 58 patients $(n=58)$, where the mean backache score was 2.30 and mean serum creatinine value 2.77. The Pearson $\mathrm{R}$ value obtained was 0.01 which is indicating almost no relationship between backache and serum creatinine. Patients with stage 3 kidney disease $(n=14)$ had an average E-GFR of 38.0, stage $4(n=38)$ with 22.3 and stage $5(\mathrm{n}=6)$ with 14.0.

Conclusion - Backache can be used an indicator of CKDu since it is presented in most patients' suffering from the disease but unable to be used to determine the stage of CKDu suffered by the patient due to the external causes that leads to backache. Several predisposing factors such as temperature, water source and agricultural activity can influence the backache.

Keywords - ACR- Albumin to creatinine ratio; BUN - Blood urea nitrogen; CKD - Chronic Kidney disease; E-GFR - Estimated glomerular filtration rate; ESRD - End stage renal 
disease; MDRD - Modification of Diet in Renal disease; MOH - Ministry of Health; NCD Non-communicable diseases; NCP - North Central Province; NSAID's - Non-steroidal antiinflammatory drugs; NWP - North Western Province; WHO - World Health Organization.

\section{List of Figures}

Figure 1: CKDu centres and most affected regions in Srilanka as adapted from Abraham (Abraham et al., 2016). 6

Figure 2: E-GFR equation as adapted from Saunders (Saunders, 2007). ........................... 8

Figure 3: Backache assessment over a period of five months...................................... 12

Figure 4: Serum creatinine assessment over a period of five months with the standard deviation and error bars 13

Figure 5: Pearson R correlation test, type of backache (x-axis) and serum creatinine (y-axis). 13

Figure 6: E-GFR estimation on average for five months. 14

\section{$\underline{\text { List of Tables }}$}

Table 1: Staging of chronic kidney disease based on E-GFR (Saunders, 2007)................... 3 Table 2: Types of backache assessment carried out on chronic kidney disease patients as adapted from Kafkia (Kafkia et al., 2011) ..................................................................... 10

Table 3: Monthly data for the analysis carried out between November to April. ................ 11

Table 4: E-GFR estimation and type of risk associated using MDRD equation.................... 14 


\section{Chapter 1: Background}

Kidney disease is a prominent disease which is well spread among Srilankan population, there are two main types of kidney disease, chronic kidney disease (CKD) and chronic kidney disease of unknown aetiology $(\mathrm{CKDu})$. Chronic kidney disease patients have common symptoms such as hypertension and diabetes that affects them, these can be used an indication for the patient developing CKD. Unlike CKD, CKDu has no symptoms and this is an irreversible disease, many factors are linked to causing the disease in patients' mainly from the agricultural regions of Srilanka (Redmon et al., 2016).

According to an article published by Rajapakse in 2016, a prevalence of $15.1 \%$ to $22.9 \%$ $\mathrm{CKDu}$ is presented in districts associated with agriculture where most people undertake farming as an occupation. A reported 69258 patients were diagnosed with CKDu in Srilanka, 2015 as stated by Abraham, out of which the North Central Province districts Anuradaphura and Polonnaruwa are the most disease prone regions with a reported 50382 cases being identified. Diagnosis of CKDu is hard during the initial stages since the body shows no signs or symptoms presented, renal biopsy will indicate tubulointerstitial fibrosis or tubular atrophy in the later stages as stated by Redmon et al., (2016). Using E-GFR the diagnosis can be made early and the type of CKDu can be analyzed and the levels of proteinuria, also known as albumin to creatinine ratio can be used to distinguish the stage of $\mathrm{CKDu}$ in the patient (Abraham et al., 2016; Rajapakse, Shivanthan and Selvarajah, 2016).

\section{$\underline{1.1 \text { Scope }}$}

The project focuses on identifying the relationship between serum creatinine and backache presented in the patients. This allows us to identify causes that have not been detected in cases of $\mathrm{CKDu}$ which allows an early diagnosis to be carried out that helps the society and benefits the researcher to identify various causes leading to backache in these patients. 


\section{Chapter 2 - Literature Review}

\subsection{CKDu in Sri Lanka}

$\mathrm{CKDu}$ is commonly endemic in regions of North Central Province and the main factor that associated increased rates are the high temperature in the dry zone and agriculture in which the farming elderly population are relatively higher (Jayasekara et al., 2013). Various causes have been identified from 2009 onwards where the CKDu was recognized as a burden by Ministry of Healthcare and Nutrition in Srilanka (Senanayake et al., 2017)

Fluoride and heavy metals are one of the main causes of this presented in patients in NCP due to the water source predominantly that contains fertilizer heavy metals used in cultivation (Wanigasuriya, 2012).

A common symptom that is associated with renal failure is backache where patients suffering from kidney disease has this commonly in them. Backache can be used as an indirect marker that analyses the pain presented by the patient for a period of days, weeks, months or years to identify the stage of the disease.

\subsubsection{Role of kidney in the human body and the function.}

Kidney is a key organ presented in the body that is involved in the urinary system of the body, these are bean shaped organs which function as a unit to regulate homeostasis which maintains the environment constantly inside the body. The hormone erythropoietin is also produced by the endothelial cells of the kidney which causes production of red blood cells. The excretory waste substance also known as urine is produced by the kidney by a series of events which ensures the waste material is removed out of the body into the filtrate (Hemmelgarn et al., 2010).

Damage to the kidney can result in developing into end stage renal disease (ESRD) which can cause permanent damage to the kidneys. Several complications can arise due to the kidney damage in the patient such as problems in urine production which causes toxic substances building inside the body, this can cause weakness, body pain and swelling in the patient. Another severe complication is the collection of gall stones, this can cause urinary tract infections in the patient leading to infections spreading to the kidney and causing long term permanent damage. Oxygen imbalance is another complication that can occur where the hormone erythropoietin involved is unable to be produced when required leading to 
symptoms such as poor appetite, difficulty sleeping, shortness of breath and frequent late night urination (Bhatt et al., 2014)

Srilanka is mainly affected by the two forms of kidney disease, they are CKD and CKDu.

\subsubsection{Chronic Kidney disease}

Chronic kidney disease also known as CKD is a prominent form of kidney disease that affects the global population. The main chronic diseases that causes CKD are diabetes mellitus and hypertension cited by the United States Renal Data System in the article published by Gunatilake in 2015. This is a progressive disease that cannot be diagnosed until the disease progresses into its fatal stages, these patients will show symptoms associated with the disease that can be treated when diagnosed early. People who are older than 50 have the most likely tendency to develop into $\mathrm{CKD}$, this can cause the patient to develop cardiovascular diseases as well (Jayathilake et al., 2013; Gunatilake, Samaratunga and Rubasinghe, 2015).

CKD can be differentiated on two main pathways, kidney damage which can be detected by proteinuria levels by calculating the albumin to creatinine ratio (ACR) or else decrease in kidney function that can be detected using the e-GFR. CKD can be differentiated into five stages based on the GFR levels obtained in the patient.

Table 1: Staging of chronic kidney disease based on E-GFR (Saunders, 2007).

\begin{tabular}{|l|l|l|}
\hline Stage & E-GFR $/ \mathrm{ml} / \mathrm{min} / 1.73 \mathrm{~m}^{2}$ & Kidney Condition \\
\hline 1 & $\geq 90$ & Normal \\
\hline 2 & $60-89$ & Mild decline \\
\hline 3 & $30-59$ & Low risk of developing kidney failure \\
\hline 4 & $15-29$ & High risk of developing kidney failure \\
\hline 5 & $<15$ & Kidney failure \\
\hline
\end{tabular}

Since the disease is asymptomatic the patient will not show any signs of discomfort until reaching the $4^{\text {th }}$ or $5^{\text {th }}$ stages. Males are predominantly affected more in the severe stages compared to females. The staging of CKD determines the kidney disease type and treatments 
can be given depending on the stage classified. Patients suffering from CKD have the likelihood of developing into end stage renal disease (ESRD) if left untreated, this can cause serious complications in the patient resulting in dialysis or kidney transplant being the only option available. According to Abraham, 1.8/1000 population in an affected region is affected by CKD and overall 0.4 people in a population of 1000 are affected by CKD in Srilanka (Abraham et al., 2016).

According to the Global burden of disease study carried out in 2010, around $10 \%$ of the world population is affected by CKD, a reported 271.8 million women and 225.7 million men were affected. In 1990 the disease was ranked at $27^{\text {th }}$ in the total number of reported deaths which climbed eight places to $19^{\text {th }}$ in 2010 only second to the rise of HIV within this time period (Mills et al., 2015).

In America around 30 million people are affected by CKD according to the 2017 national chronic kidney disease fact sheet. $\mathrm{CKD}$ has contributed significantly making noncommunicable diseases the common cause of premature death around the world out of which $75 \%$ of Srilankan population are affected by NCDs (Centers for Disease Control and Prevention, 2017)

\subsubsection{Chronic Kidney disease of unknown aetiology}

Chronic kidney disease of unknown aetiology also known as CKDu was initially observed in Srilanka during the 1990's. CKDu is a slow progressive disease like CKD without the symptoms and this cannot be controlled since the disease is irreversible. The most common provinces afftected by CKDu have been listed as the North Central Province (NCP), Uva Province (UP) and Eastern Province (EP) (Wanigasuriya, 2014).

$\mathrm{CKDu}$ affects the proximal tubules of the kidney, the normal function of the proximal tubule is to regulate reabsorption of the filtrate substances and it acts as an immune response during immunological or toxic injuries in the kidney. Damage to the proximal tubule also causes the kidney to reduce erythropoietin synthesis which is needed for red cell production, this can lead to patient having chronic kidney disease (Wijethunge et al., 2015).

Most commonly people aged between 40-60 and having an occupation related to agriculture are the most liable people to have the disease. 
$\mathrm{CKDu}$ is diagnosed using the proteinuria levels and E-GFR and can be categorized into four stages, the symptoms are not presented by the patient until reaching stage 3 or 4 making the diagnosis and treatment difficult.

In Srilanka the NCP is considered an endemic region for $\mathrm{CKDu}$, this is due to main occupation being farming in the region, according to Agriculture and Environmental statistics division for 2017 Anuradaphura and Polonnaruwa produces $19.53 \%$ of the paddy in the country. Chena cultivation also influenced the likelihood of the person developing CKDu where the farmer shifts lands to grow vegetables or other crops other than paddy. The overall population affected mostly are the females but the most severe stage 3-4 males are affected more. The patients suffering from final stages 3-4 showed interstitial fibrosis presented in them when a renal biopsy was taken (Wijekoon, Perera and Herath, 2017)

Other countries such as Mexico, El Salvador, USA and India also high number of reported cases of CKDu according to the article published by Weaver in 2015. El Salvador has the highest ratio of CKDu prevalence where 85.5 men out of a given population of 1000 have the disease and women with 34.1, comparatively Srilanka has a prevalence only in districts such as Anuradhapura, Polonnaruwa and Badulla making up 58.6\% of the reported cases in Srilanka (Weaver, Fadrowski and Jaar, 2015).

Many factors have been associated with the prevalence of CKDu in Srilanka, a research carried out by the MOH and WHO from a period of 2009-2012 in Srilanka found out several factors that can influence the chances of CKDu being present in a person. Agriculture has been listed as one of the main problems since the soil used contains a lot pf heavy metals such as arsenic, cadmium and phosphorous are present although there is no clear evidence that they cause the disease, hypothesis have been built to support the facts. Geographical characteristics also play a part where the NCP is very dry and only two monsoon seasons are available in the region with a rainfall of $1000-1500 \mathrm{~mm}$ every year. Water source is another factor since heavy metals can remain the water supply which can be taken while drinking and also while food preparation, the presence of ochratoxin A contains nephrotoxins that can damage the nephrons of the kidney (Wijethunge et al., 2015).

A research brief carried out by Elledge, only a reported 8000 personals are undergoing dialysis treatment due to the restrains in socio-economic status presented. Many patients who 
are unable to pay for the treatment remain in their severe states and eventually are leading themselves to death due to this. Srilankan government hospitals only contain a reported 25 hemodialysis centers offered by the government which also is a limitation since private sectors charges are relatively high at $\$ 65$ for a period of 4 hours (Elledge et al., 2014).

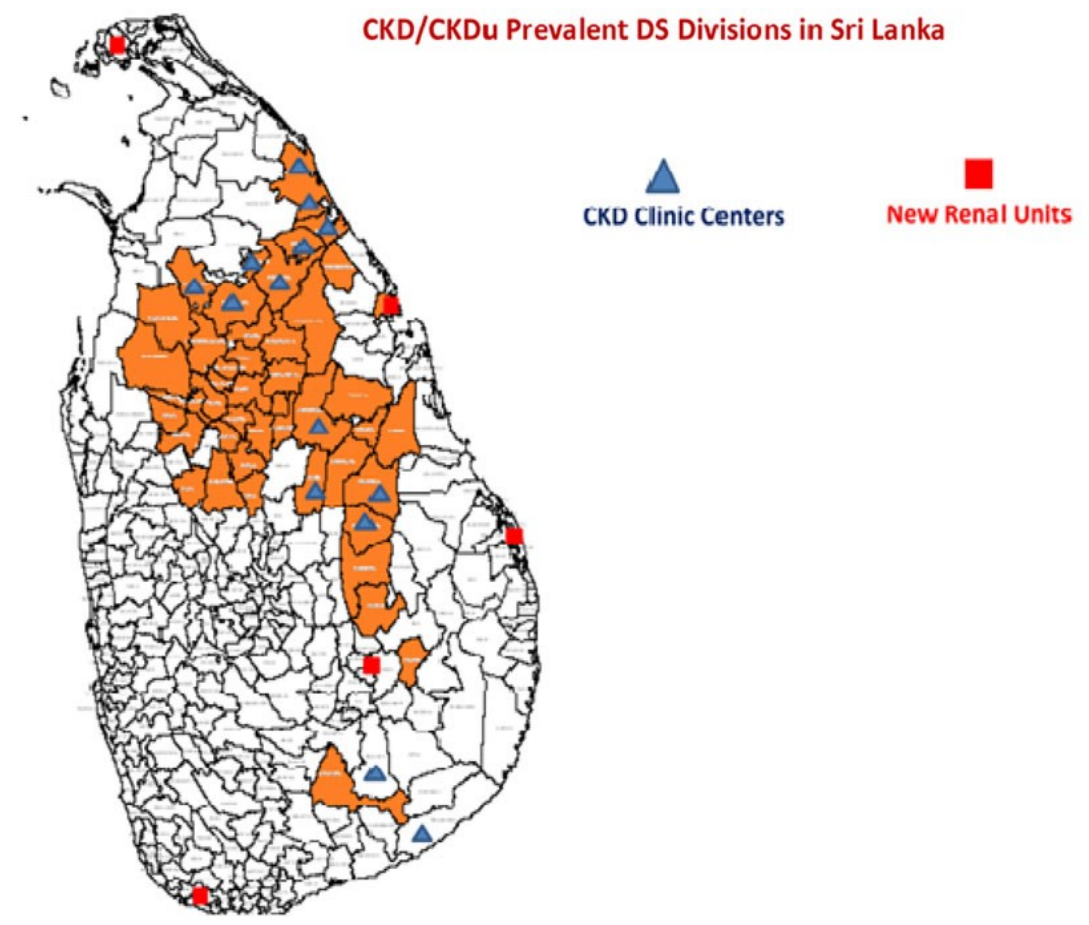

Figure 1: CKDu centres and most affected regions in Srilanka as adapted from Abraham (Abraham et al., 2016).

\subsubsection{Creatinine levels as a Bio-marker for kidney disease}

Creatinine is a breakdown waste material produced by the creatinine phosphate present in the body, in normal kidney the serum creatinine produced by the body is on average of $0.5-1.2$ $\mathrm{mg} / \mathrm{dL}$ for males and $0.4-1.1 \mathrm{mg} / \mathrm{dL}$ for females. This reference range can vary depending on the BMI, age, ethnicity and race since various population have different amounts of serum creatinine presented in their body.

Creatinine is absorbed into the body by the gastrointestinal tract mainly by the ileum and jejunum presented, and distributed mainly towards the skeletal muscles. This is initially presented as the molecule creatine which is converted into phosphocreatine. Creatine to creatinine conversion rate is a major factor that determines the GFR rate in the patient and the 
body temperature also determines the amount of creatinine present since at high temperatures the solubility is higher in the body (Salomons and Wyss, 2007).

Creatinine is excreted out of the body by the glomeruli and proximal tubules of the kidney, according to Stevens (2006), this can be used to estimate glomerular filtration rate since no tubular reabsorption takes place in creatinine clearance. Using the serum creatinine values the progression and staging of $\mathrm{CKDu}$ can be identified and the appropriate treatment can be given to the patients based on the results obtained (Stevens et al., 2006).

\subsubsection{Estimating glomerular filtration rate (E-GFR)}

According to the Kidney foundation disease outcomes quality initiative (KDOQI), GFR is used to classify chronic kidney disease in the patient. This initiative was implemented in 2004 and several changes have been taken in order to ensure that the classification and staging is carried out in an accurate manner to ensure the proper diagnosis to be given to the patient. The criteria of diagnosis a patient with chronic kidney disease requires a e-GFR $<60$ $\mathrm{ml} / \mathrm{min} / 1.73 \mathrm{~m}^{2}$ for a period of greater than three months (Saunders, 2007).

Estimating GFR can be done by using the filtration markers such as serum creatinine or cystatin C most commonly as stated by Lopez-Giacoman. GFR is the fluid filtration volume from glomerulus capillaries to Bowman's capsule per unit time and is dependent on the body mass index, muscle mass, age, gender and ethnicity mainly. Creatinine has a low molecular weight of $113 \mathrm{Da}$ making it easily measured as it is freely available in the body (LopezGiacoman and Madero, 2015).

The most common equations used to estimate GFR are MDRD (modification of diet in renal disease) and CKD-EPI (chronic kidney disease epidemiology collaboration). CG equation was implemented by Cockcroft and Gault in 1976, although the creatinine clearance was measured it was less reliable and the equation was less used with time. MDRD equation was found out in the study that was carried out in 1999 with the variables being age, sex, ethnicity and serum creatinine. This is considered one of the most accurate tests for CKD patients but also could result in false-positive test results presented (Florkowski and Chew-Harris, 2011). CKD-EPI is another equation which is considered as an alternative for the MDRD, this is most recent discovery where a study carried out in 2009 lead to the discovery of the equation. 
This equation can be used in patients without CKD or suspected to have since less chances of false positive diagnosis to be carried out and the CKD prevalence is lower using the CKDEPI equation (Florkowski and Chew-Harris, 2011).

Latest researchers have found out equations that will provide more accurate readings compared to CKD-EPI and MDRD. A combination of CysC and serum creatinine levels, this provided accurate diagnosis of the patient with or without CKD according to the study carried by Chehade (Chehade et al., 2013).

MDRD equation;

eGFR $\left(\mathrm{mL} / \mathrm{min} / 1.73 \mathrm{~m}^{2}\right)=186 \times(\mathrm{SCr})^{-1.154} \times(\mathrm{Age})^{-0.203}$

$\times(0.742$, if female)

$\times(1.212$, if African American)

Replace the constant 186 with 175, if the laboratory uses a standardized SCr (IDMS method). This reduces eGFR by $6 \%$.

Figure 2: E-GFR equation as adapted from Saunders (Saunders, 2007).

\subsubsection{Backache in patients with Chronic kidney disease with unknown aetiology}

Backache is a prominent factor presented in the patient where the kidney failure results in waste and toxic build up inside the body. According to literature review carried out by Davison in 2010 a reported $49 \%$ of the patients were presented with pain either moderate or severe forms (Davison and Jhangri, 2010).

The quality of life in patients suffering with CKD has been rated poor by Jung Hsu and team of researchers in a cross sectional study carried out in 2014. Musculoskeletal pain has been identified as the most common symptom presented in the patient where the main pain arises from the places of movements in the body such as joints, posture balance and bone movement presented in the patient. Due to the chronic kidney disease the patient develops musculoskeletal disorders in the body that will cause body support and movement problems. (Hsu et al., 2014). 
Backache is predominantly associated with the musculoskeletal pain since the spinal cord is needed for the movement and balance of the person's body, this is identified as a common symptom associated with chronic kidney diseases. Due to this the patient will have problems in carrying out day to day work and sleep issues are commonly presented in the patient (Hsu et al., 2014).

According to an article published by Kafkia the pain is found between the "twelfth thoracic and the third lumbar (T12-L3)" in the patient. Depending on the symptoms, duration and depth of pain these can be categorized into several types (Kafkia et al., 2011).

According to the WHO step by step approach for chronic pain, the patient can undertake the necessary treatments presented in them, although medications are taken if too much of drugs are taken by the person for pain relief he/she can end up in a state of toxicity ensuring further damage to the kidney presented. The most common types of drugs used are paracetamol, NSAID's are commonly used to rest the symptoms in the patient.

Pain in the patient can significantly reduce the quality of life since the person affected with pain is taken into a series of wild thoughts of depression and considering the disease as a burden for their lives. This can lead to problems associated with carrying out day to day activities related to life, including sleeping and mental issues presented in the patient.

\section{Chapter 3: Methods}

\section{$\underline{3.1 \text { Interview based questionnaire }}$}

The patients will be questioned using a questionnaire designed by the Kothalawela Defense University which is approved by the Institute of Biology (Sri Lanka). It only focuses on the questions related to pain and quality of life of the patient to minimize the number of questions and obtain a proper scale based on the obtained information. Common questions related to backache such as, how long is pain prominent? Difficulties in carrying out day-to-day activities are common questions asked from the patients. The study carried out complies with all regulations and confirmation that informed consents were obtained from the patients. 


\subsection{Grading scale}

After analyzing the questions answered by the patients, the symptoms and the duration of pain will be used to assess the type of pain presented in the patient. Based on the pain classification table adapted from Kapkia's research article the pain is assessed into four main types, neuropathic, nociceptive, intermittent pain and no backache. A scale of scores from 1-4 is given to the patients with the least being for no backache and highest for the neuropathic pain observed.

Table 2: Types of backache assessment carried out on chronic kidney disease patients as adapted from Kafkia (Kafkia et al., 2011)

\begin{tabular}{|c|c|}
\hline Acute pain & $\begin{array}{l}\text { Pain of recent onset that is usualy transient in nature, bating from several minutes to several deys or weets. Caused by tissue damage and is } \\
\text { ofien associobed with some degree of inflammation. }\end{array}$ \\
\hline Chronic pain & $\begin{array}{l}\text { Pain that perxists beyond the usual course of an acute il hess or injury (usully bejond } 3 \text { manths), ansociated with a patten of recurnence orer } \\
\text { months or years or associated with a chronic pathological process. }\end{array}$ \\
\hline Neuropathic pain & $\begin{array}{l}\text { Pain attrbuted to a peripheral injury of the nervous system or the central nervous sptem. Destruction, infitration and compreasion of the neve } \\
\text { tisue are the causes of pain. }\end{array}$ \\
\hline Nociceptive pain & $\begin{array}{l}\text { Pain is caused by dinect of mubfion of peripheral nocioeptors asocisted with tissue injury as well } x \text { inflammstion but may also be excited by } \\
\text { endogenous chemical substances. }\end{array}$ \\
\hline Constant pain & Continuing pain that does not ceave or let up but confinues for lang periods of time \\
\hline Sharp pain & Intense, severe pain \\
\hline Intermittent pain & Pain occurring occasionally or at regulat or integular intervis; periodic pein. \\
\hline ISispathic pain & Pain of unlinown cause or spontaneous arigin. \\
\hline
\end{tabular}

\section{$\underline{3.3 \text { Blood collection and analysis }}$}

The blood sample obtained is centrifuged initially at 3000rpm for five minutes, the serum sample is separated using micro-pipette that is calibrated with a blank sample before using. Callibration is carried out to ensure that accuracy of the sample measurement using pipette process takes place and to minimize the errors caused during analytical phase (WHO, 2012). Serum is transferred onto an Eppendorf tube and labelled according to each patient serial number.

\subsection{Serum creatinine analysis}

Serum creatinine is measured using the blood sample centrifugation in which the plasma is separated from the blood cells and then stored at around -20 degrees Celsius and then analyzed using a biochemical analyser. Serum creatinine levels using Jaffe's reaction is determined in this analyser by using the chemicals picric acid and sodium hydroxide presented in the solution with acetic acid which acts as the alkaline medium. This method is one of the oldest method being used in the $21^{\text {st }}$ century as well since it is easy to use and cost efficient (Delanghe and Speeckaert, 2011). 


\subsection{E-GFR calculation using creatinine}

E-GFR is calculated using the MDRD equation implemented in 1999, using the E-GFR equation the staging of chronic kidney disease can be assessed in the patient (Florkowski and Chew-Harris, 2011). To support the E-GFR the proteinuria levels can be identified by calculating the ACR (albumin to creatinine ratio). Albumin can be calculated using the sample from urine, in which the urine sample is analyzed using immune-turbid metric assays. The results are analyzed over a period of five months, with variation in backache and serum creatinine levels being checked. Collected data is analyzed using Pearson R correlation test that will determine if there is a relationship between backache observed and serum creatinine levels. This is done using IBM SPSS 23 and graph pad prism 7, in which correlation between the results are observed.

\section{Chapter 4: Results}

Serum creatinine and backache were observed for a period of five months and using this obtained set of data the Pearson R correlation value is calculated. Over a hundred individuals were assessed and evaluated during the grading of the backache, out of which 58 patients only visited five consecutive clinics from November to April.

\section{$\underline{4.1 \text { Variation in Overall Results over the period of five months }}$}

Table 3: Monthly data for the analysis carried out between November to April.

\begin{tabular}{|l|l|l|l|l|l|}
\hline Monthly Data & $1^{\text {st }}$ Month & $2^{\text {nd }}$ Month & $3^{\text {rd }}$ Month & $4^{\text {th }}$ Month & $5^{\text {th }}$ Month \\
\hline Pearson R value & 0.07 & 0.09 & -0.11 & 0.16 & -0.20 \\
\hline Mean Backache Score & 2.28 & 2.59 & 2.05 & 2.50 & 2.09 \\
\hline Mean S.Creatinine (mg/dL) & 2.80 & 2.98 & 2.23 & 2.59 & 3.18 \\
\hline Number of samples (N) & 58 & 58 & 58 & 58 & 58 \\
\hline
\end{tabular}

As mentioned in table 3 the Pearson $R$ values obtained show both a negative and positive variation in between the five months. This indicates that the serum creatinine is an independent factor that varies accordingly to different types of backache presented. 


\subsubsection{Backache variation over five months.}

Backache was evaluated using a standard questionnaire and selected questions based on dayto-day activity to evaluate backache. Using the responses obtained from the questions the backache is determined using Kafkia's research article based on backache on chronic kidney patients the type of backache is determined. As listed in the above table 3, the backache score remained between intermittent and nociceptive backache presented which is between the scores of 2-3 in the patients.

\section{Average backache score obtained from questionnaire}

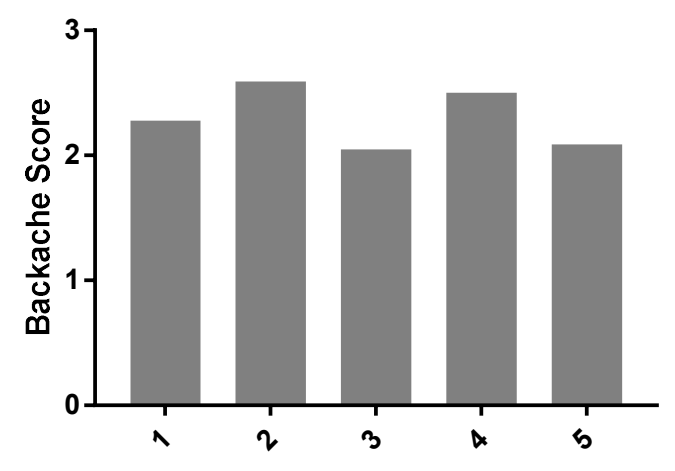

Month

Figure 3: Backache assessment over a period of five months.

\subsubsection{Serum Creatinine Variation over five months.}

Serum creatinine is an independent factor that varies with different types of backache presented. This acts as an input for the dependant variables to act on them causing constant changes. As mentioned above in the table 3, the lowest serum creatinine was observed in the $3^{\text {rd }}$ month $(2.23 \mathrm{mg} / \mathrm{dL})$ and highest in the $5^{\text {th }}$ month $(3.18 \mathrm{mg} / \mathrm{dL})$. The average serum creatinine levels differ in males and females as mentioned above. The below figure shows the variation in serum creatinine using the mean and standard deviation presented, this allows the evaluation of backache to be determined using the independent variable results. 
Average serum creatinine and variation over a period of five months

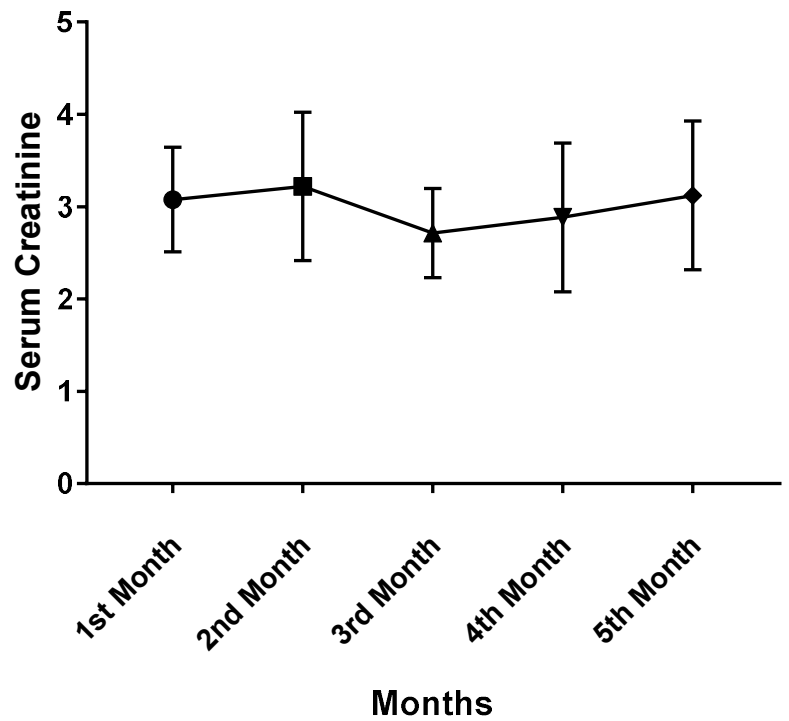

- 1st Month

tr 2nd Month

- 3rd Month

$\rightarrow$ 4th Month

$\neg$ 5th Month

Figure 4: Serum creatinine assessment over a period of five months with the standard deviation and error bars.

\subsubsection{Pearson correlation between serum creatinine and backache}

\section{Backache Correlation with serum creatinine}

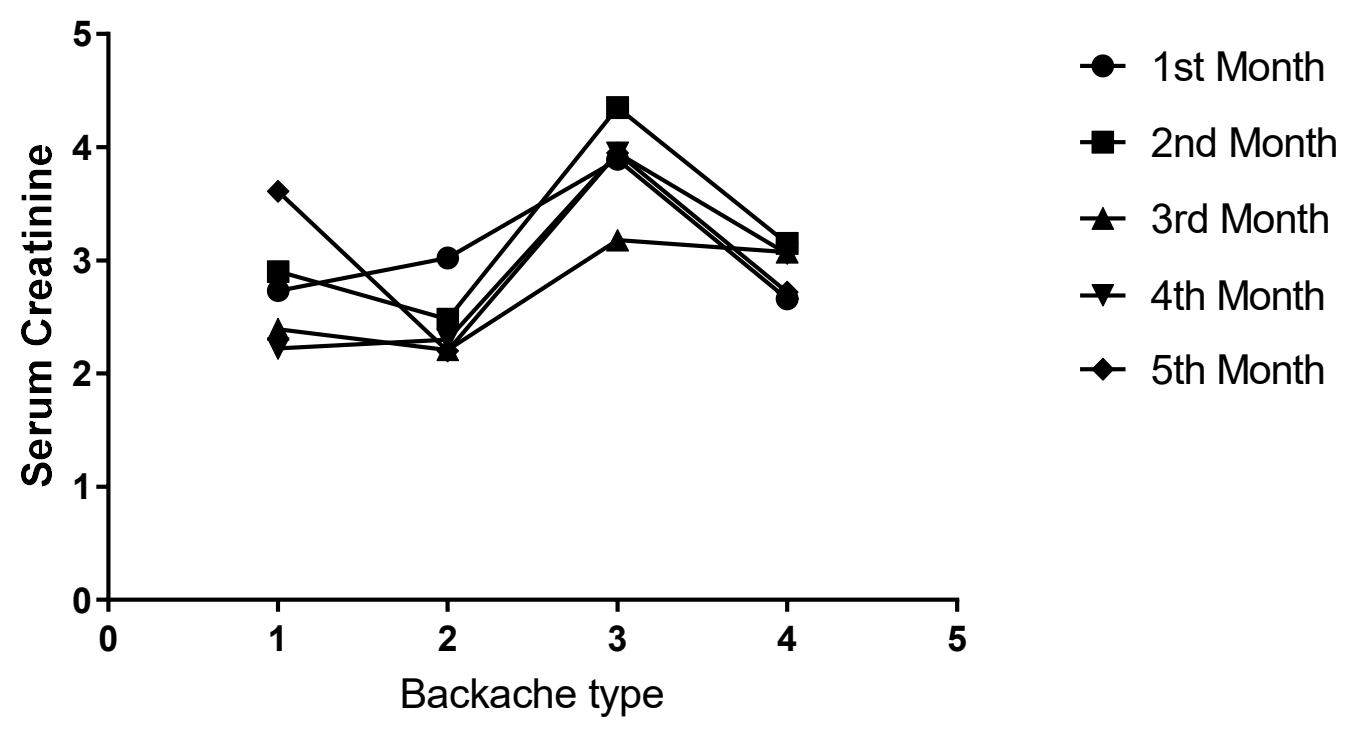

Figure 5: Pearson R correlation test, type of backache (x-axis) and serum creatinine (y-axis).

The above figure shows the Pearson correlation carried out on the initial set of results obtained, this indicates a similar trend line over the period of five months. Pearson $\mathrm{R}$ 
correlation test used allows to measure the strength of linear correlation between two variables using the standard deviation.

Backache type 1 is prominently presented in the $5^{\text {th }}$ Month patients, type 2 in $1^{\text {st }}$ month patients, type 3 in $2^{\text {nd }}$ month patients and type 4 in $2^{\text {nd }}$ and $1^{\text {st }}$ month patients.

\subsection{E-GFR Estimation}

As mentioned above in table 1, E-GFR can be categorized into five main classifications of which symptoms can be given. Using the MDRD-EGFR equation as listed above in figure 2 the calculations for the 58 patients for a period of five months on average is calculated. The below table shows the results of the patients.

Table 4: E-GFR estimation and type of risk associated using MDRD equation

\begin{tabular}{|l|l|l|}
\hline E-GFR $/ \mathrm{ml} / \mathrm{min} / 1.73 \mathrm{~m}^{2}$ & Risk of CKDu & Number of Patients affected \\
\hline $30-59$ & Low risk - Stage 3 & 14 \\
\hline $15-29$ & High Risk - Stage 4 & 38 \\
\hline$<15$ & Kidney failure - Stage 5 & 6 \\
\hline
\end{tabular}

\section{Variation in E-GFR from November to March}

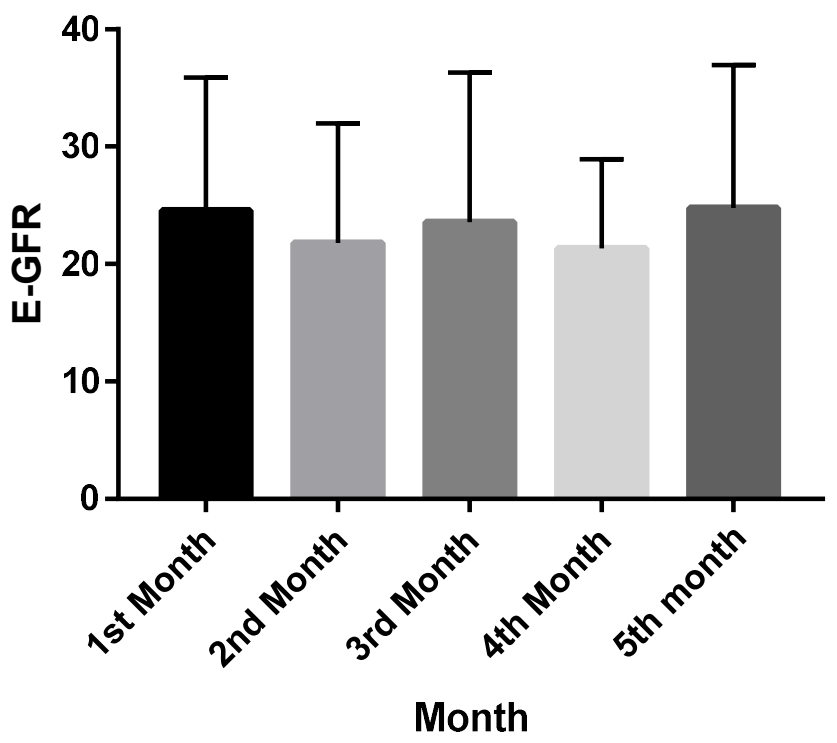
1st Month
2nd Month
3rd Month
4th Month

5th month

Figure 6: E-GFR estimation on average for five months. 
The above figure 6 shows the variation in E-GFR measurements, in which the lowest is detected in the $4^{\text {th }}$ month. The estimation of this allows to diagnose the stage of CKDu in the patients where most commonly patients were suffering from stage 2 which is most abundant stage over a period of five months. 


\section{Chapter 5: Discussion}

$\mathrm{CKDu}$ is prominent among all 58 patients analysed and tested with most of them in stage 3,4 and 5 as mentioned above in table 4. A Pearson $\mathrm{R}$ value obtained over a period of five months shows a value of 0.01 on average, this is almost close to $r=0$ making it indicating that is there no relationship between backache levels and serum creatinine.

Evaluation of backache was carried out using an interview based questionnaire which involves selected set of questions listed related to backache. This is an easy efficient process that does not consume time of patients to fill in the forms and responses are obtained by the patient at a faster rate. The right questions chosen for the assessment of backache allows reduced number of questions being asked and the correct order limits the problems that can arise asking too many questions from the patients (Gillham, 2008). The questions carried out were either opened questions, the ones with limited options such as yes and no and closed questions that draws the reply from the patient itself. Based on the replies given by the patient the results were converted into a quantitative measurement that assesses the type of backache (Rowley, 2014).

Although several advantages are presented in using a questionnaire for quantitative analysis where large number of data samples can be collected there are several limitations as well that influences the results obtained by the researcher. Most commonly the patients were not honest with giving answers leading to biased answers being presented during the interview. Misinterpretation of the question asked and lack of understanding the question can lead to confusion in the minds which causes sudden responses to be given to the researcher carrying out the survey. Certain questions are difficult to analyse so most patients tend to skip the questions presented leading to lack of responses for a particular set of questions listed by the researcher which can influence the overall statistical analysis carried on the results of the questionnaire (Saris and Gallhofer, 2014).

Backache is common in kidney disease patients of all kinds due to renal failure in these patients. Assessment of backache using questionnaires are less accurate and reliable than physical examination carried out on the patients, most commonly the COPCORD questionnaire is used to identify pain on various parts of the body and provides a scale for the results obtained. 
According to Davison (2014), 58\% of the patients suffering with kidney disease have pain presented in them and $49 \%$ of those have moderate of severe pain presented by their body (Davison, Koncicki and Brennan, 2014). Physical examination can be carried out on these patients, JOABPEQ is a standard measure used in Japan to evaluate the pain in patients by carrying out specific tasks that do not cause harm to them or hurt them in anyway. This method was implemented in 1986 and has been highly recognized throughout the world in assessing low back pain in patients which is a common sign in kidney damage (Hashizume et al., 2015).

Several patients suffering from pain also take in medications to get relief from the pain, these are most commonly analgesic used such as NSAID's, paracetamol and anticonvulsants (Manias and Williams, 2008). These drugs normally metabolize in the liver and causes increased toxic products presented inside the body circulation which can influence the renal failure to increase furthermore. Several patients do take in medications but do not accept the fact that they are taking medication, this can be one of the causes that influences the backache. Actions such as sitting, standing and awkward posture imbalance due to occupation can also influence this, patients suffering with $\mathrm{CKDu}$ are mostly farmers who undertake agriculture which involves long hours of standing and bending in awkward positions can lead to pain increasing (Balagué et al., 2012).

Chronic musculoskeletal pain is another dominant feature presented in these patients where patients with chronic kidney disease develops this pain due to intake of herbal medication mostly. This involves pain in the joints, movements and tendons most commonly and increased calcium levels presented during early stages 1-3 of CKD. Smoking and alcohol can further induce backache and MS pain presented by the patients where carcinogens and chemicals damage the liver causing increased release of toxic products that affects the renal function of the kidney (Hsu et al., 2014).

Blood collection is another important aspect of this research where using the blood sample obtained analysis of serum creatinine take place. The blood samples were collected by a phlebotomist, a venepuncture is made on the veins and the blood is drawn into a syringe which is then stored into an EDTA tube. Since the patients presented are mostly elderly population, the patients tend to have fear during blood collection and draw away the hand during collection. This can lead to contamination of the needle which can cause the blood 
drawn to have contaminants as well during analysis. Infection is another problem in which the patients are liable to get infections after the blood has been drawn due to an opening of the skin. Hematoma formation is another complication where the blood drawn can cause blood leakage into the tissues leading to swelling signs presented by the patient. One of the most common cause of low blood collection is the patient having fear during the process causing less blood flow around the region leading to lowered blood being collected (Buowari, 2013).

Once the blood has been collected the samples are kept for a period of nearly three hours before being transferred into the ice containing container. This results in high chances of blood clots being formed in the tube resulting in the cellular components being damaged and variation in serum creatinine to be observed due to this. Variation in RBC morphology and cellular components happen due to this resulting in potent toxic and waste compounds presented in the collected blood sample in EDTA tube (Van de Watering and Brand, 2008).

The samples are transferred into an ice container stored at -20 degrees Celsius and then centrifugation process takes place. Centrifugation is carried out using thermo heraeus megafuge 8 at 3000rpm for a period of five minutes. Most of the samples obtained are frozen therefore the pellets are disturbed by tapping the bottom of the tube in order to break the frozen specimen. This can lead to haemolysis due to vigorous shaking of the tube that results in increase concentrations of intracellular metabolites commonly, another factor is centrifugation at high speed can further damage the concentration of blood cells presented. The normal procedure involves collection of blood within one hour analysed at 2500rpm, since the collection period is longer in these patients the blood sample obtained is centrifuged at 3000rpm commonly. The sudden change in temperature also can influence the results obtained in the patient where from high temperatures presented to sudden low temperature can cause in increased metabolites (Yin, Lehmann and Xu, 2015).

Quality control is an essential component that is needed for the right tests and procedures to be carried out in the laboratory. The use of standard operating procedure (SOP) can result in avoiding or minimising errors formed by the blood drawing and collection process, most commonly during pre-analytical stage labelling the tubes is the most common error. Eppendorf tubes were not labelled properly resulting in errors during marking and transferring of the serum samples into new tubes (Rana, 2012). 
The serum samples were then stored back into the ice container to avoid deterioration of the sample; this was analysed using an automated analyser later. Serum creatinine is released as a metabolic waste product which has a half-life of 40 days in normal patients with normal body $\mathrm{pH}$ values, an increase in $\mathrm{pH}$ values can reduce the life span of creatinine. Due to renal failure patients $\mathrm{pH}$ changes due to fluid imbalance as damage to the kidney takes place. Creatinine can also be lost in faeces and urine making it difficult to identify the range of creatinine presented by the body (Salomons and Wyss, 2007).

The samples collected were analysed after a long period of time after freeze thawing taking place where the samples were stored at relatively high temperatures. This can cause the creatinine levels obtained to have variations than the original values causing the reliability of the results to be affected by $\pm 10 \%$ generally and could be higher if kidney damage is presented (Salomons and Wyss, 2007).

Jaffe's reaction is a method that has been used for more than a century commonly for the measurement of creatinine using colorimetric analysis under the spectrometer. This method involves creatinine mixed with picric acid and dissolved in an alkaline solution of acetic acid presented and using the colour change observed at a specific wavelength the sample creatinine levels are detected. This is still being used since it is simple and can be carried out at a lower cost, although there are advantages using this method several factors can cause results changes to occur (Delanghe and Speeckaert, 2011).

Most commonly the colour change in the complex formed varies over time resulting in varied results being obtained for the patient sample. Another factor that influences the serum creatinine value in Jaffe's reaction is the wavelength the results are obtained can vary based on the colour change of the complex influencing differences in reactions. Intake of drugs can influence the false positive elevation of creatinine levels most commonly and since $\mathrm{CKDu}$ patients are taking in regular medications this can affect the creatinine results (Syal, Banerjee and Srinivasan, 2013).

Enzymatic Jaffe's reaction and modified Jaffe's reaction are the most advanced techniques used to minimize the problems caused by the traditional methods. Isotope dilution mass spectrometry (IDMS) is a method that has been implemented to minimize the variations. This involves revised MDRD equation instead of using 186 as stated above in figure 2, 175 is used 
this is because the recalibration of the creatinine measurement is taken place where a $10 \%$ error in minimized in E-GFR calculations. Roche Hitachi 917 is the most widely used automated machine that uses a blank sample and second sample to compare and differentiate the enzymatic assays of creatinine (Peake and Whiting, 2006).

Several other biomarkers have been considered as an alternative to creatinine since it is produced by the body and a small amount of creatinine is excreted as well, people with large body muscle mass tend to have higher creatinine levels making the use of creatinine as a biomarker less reliable.

Cystatin $\mathrm{C}$ is widely considered as an alternative marker since it is reabsorbed by the body and does not undergo tubular secretion like creatinine used in the E-GFR measurements. It is also closely correlated to E-GFR values obtained and can be used as a diagnosis tool for preclinical kidney disease in the patient (Shlipak et al., 2005). Blood urea nitrogen is another indicator which has a negative feedback on GFR values where an increase in BUN indicates decrease in GFR levels (Lopez-Giacoman and Madero, 2015).

CKDu has been a burden in Srilanka over the past two decades mainly in the NCP and NWP where $64.5 \%$ are in the stages 4 and 5 of the disease (Abraham et al., 2016). People aged over 40 are the most liable to get this disease since most of the elderly population carries out the farming process and most commonly affects males than females. By the time of diagnosis is made the patient is most likely to reach end stage renal disease (ESRD) since this is asymptomatic disease that is irreversible once caused. Dialysis is the only way of treating the patient but due to the high cost of treatment farmers refrain from undergoing dialysis regularly causing the symptoms to grow further in them (Noble et al., 2014).

Several factors influence the prevalence of $\mathrm{CKDu}$ in $\mathrm{NCP}$, one of the main factors that is commonly associated with the disease is the use of agrochemicals as fertilizers in agriculture. Agrochemicals act on the crops with heavy metal content, most commonly the farmers have direct exposure to this as most of the fertilizers are dispersed into the soil by bare hands. This can enter the body directly by any opening or wound present or else indirectly through the volatile particles presented in the atmosphere being inhaled or the soil eroding causing the soil to mix with the surface water running into the main flow of water supply (Redmon et al., 2016). 
Most common heavy metals include arsenic, cadmium, aluminum, fluoride and lead which can lead to water hardness being presented in the water consumed. Increased arsenic, lead and cadmium concentrations from inorganic fertilizers most commonly can lead to CKD in most patients, these are chronic toxins that can enter the body through ingestion and inhalation (Gunatilake, Samaratunga and Rubasinghe, 2015).

Cadmium is considered to be the most important heavy metal that causes the disease in the patient where most commonly consumed in the form of groundwater. This causes an increase in biomarkers such as beta-2-microglobulin and urinary albumin concentrations that leads to increased risk of developing nephrotoxicity inside the body causing kidney injury (Wanigasuriya, Peiris-John and Wickremasinghe, 2011).

Fluoride is another causative element that can be present in sources such as the well and streams that run down with the soil growth. This can combine with aluminium in the water storing pans and pots and form an aluminium fluoride complex that can pass through the cell membrane and cause variation in calcium and sodium levels inside the body (Rajapakse, Shivanthan and Selvarajah, 2016).

Use of pesticides is another factor that causes increased minerals presented in water which is consumed in NCP, this causes increased phosphate production that leads to acute kidney injury most commonly which can develop into chronic with time (Valcke et al., 2017). 1naphthol and 2-naphthol are the most commonly presented molecules in the pesticides.

Stress and temperature have been identified as external causes that can increase the likelihood of developing renal nephropathy that can affect the body. Most commonly the high temperatures presented in NCP where annually only $3.6 \mathrm{~mm}$ to $50 \mathrm{~mm}$ rainfall is common in this region (Redmon et al., 2016). This can lead to dehydration presented in the body due to increased humidity in the surrounding leading to heat induced stress nephropathy presented in the patients (Herath et al., 2018).

Increased alcohol consumption and cigarette consumption can act as predisposing factors in which the carcinogens and the chemicals released by the body can result in liver failure and 
oxygenated blood supply in the body decreases resulting in kidney unable to function properly and excrete the waste samples out of the body (Redmon et al., 2016).

Genetic predisposition can interfere with the kidney disease in the patient where the environmental factors and other causative agents like heavy metals can cause genotypephenotype changes which results in the patient susceptible to develop conditions such as type 2 diabetes and hypertension most commonly (Keller et al., 2012).

Treatment for the disease is not found till now but the use of dialysis has been encouraged in most cases, according to Abraham et al., (2016) the government sector of Srilanka have 186 dialysis machines whereas the private sector contains 65 machines. Due to the increased cost of treatment people prefer alternative treatment mainly involving medications for relieving symptoms.

Ayurveda medicine is widely used on a large scale and this research carried out patients using Ayurveda medications as a treatment for the symptoms and most of the patients have benefitted. Symptoms such as increased creatinine levels, albuminuria and blood pressure are controlled using this but since these medications act directly on the kidney and liver it can increase the chances of the patient being ill with renal damage most commonly (Patel, Gupta and Patel, 2011).

Preventive measures have been undertaken the past few years to minimize the risks of CKDu being present in the country where most commonly the WHO and Ministry of Health in Srilanka have identified measures to reduce the number of reported cases of CKDu being presented (Elledge et al., 2014). Reduction in consumption of plain tea with high sugar content, refraining from using ground water or use of filters to drink water have been highly recommended (Abraham et al., 2016). Use of protective equipment such as gloves, covered shoes and face masks during addition of fertilizers minimizes the risks of inhaling the toxins presented and preventing overuse of fertilizers can limit the damage caused in the patient reducing the chances of getting CKDu (Wimalawansa and Wimalawansa, 2014). 


\section{Chapter 6: Conclusion}

The study carried out showed increased serum creatinine in most patients and backache as a common symptom in most of the patients. A larger study carried out on a larger set of people greater than the amount observed with provide better results and assessing backache using physical assessment would help to determine the association between backache and serum creatinine.

\section{$\underline{\text { Abbreviations }}$}

ACR-Albumin to creatinine ratio; BUN - Blood urea nitrogen; CKD - Chronic Kidney disease; CKD-EPI - Chronic Kidney disease - Epidemiology Collaboration; CKDu Chronic kidney disease of unknown aetiology; COPCORD - Community acquired program for the control of rheumatic disease; E-GFR - Estimated glomerular filtration rate; ESRD End stage renal disease; IDMS - Isotope dilution mass spectroscope; JOABPEQ - Japanese orthopaedic association back pain evaluation questionnaire; MDRD - Modification of Diet in Renal disease; $\mathrm{MOH}$ - Ministry of Health; NCD - Non-communicable diseases; NCP North Central Province; NSAID's - Non-steroidal anti-inflammatory drugs; NWP - North Western Province; WHO - World Health Organization.

\section{Ethics approval and consent to participate}

The ethical approval was obtained from Institute of Biology, Sri Lanka where the analysis of patient results was given approval. The consent forms were given to patients' involved in the study where they signed reading to all conditions of the research. The patients had the right to leave the study at any time willingly if they needed to. Ethical Approval - Institute of Biology, Sri Lanka - ERC IOBSL147 0716

\section{Consent for publication}

All the personal involved in the research gave their consent and agreed upon publishing this article with no conflicts of interest.

\section{Availability of data and material}

The data is available only to the researchers involved in the study and doctors who are part of the study in the CKDu-Care unit. Results will not be shared under circumstances the confidentiality of the results will be kept safely.

\section{Competing Interests}

None of the researchers have relationships with organizations that have interest over the manuscript data available. 


\section{Authors Contribution}

Dr.Nishantha Kumarasinghe initiated the idea of carrying out the project, in which CKDuCare is an organization that helps the people who have been suffering CKDu with free medical check-ups provided. With the help of several doctors who have specialized in Ayurveda Medicine this project was carried out. All the authors involved in the project read and approved the manuscript.

\section{$\underline{\text { Acknowledgements }}$}

CKDu-Care research members: Fahim Aslam, Nishantha Kumarasinghe, Lahiru Sandaruwan, Isuru Abeykoon, Dr.Piumi, Dr.Aloka Lankeshwari, Dr.Madushani, Captain Buddhadasa, Charith, Isuru, Rukshan, Fathima Rizhka, Sandun Anuradha, Chathuri, Raveena Gajanayake, Harindran, Koshila.

BCAS Staff; Fernando WSM, Dr.K.M. Ariff, Hashan Kulasena, Shazma Hiflan

Funding

The funding is provided by British College of Applied Studies, as a student of the institute the amount needed for the project is provided from the overall charges of the course.

\section{$\underline{\text { Author Details }}$}

Fahim Aslam ${ }^{1}$, University of Wolverhampton, School of Sciences, Dr.Nishantha Kumarasinghe $^{2}$, Kothalawela Defence University.

\section{$\underline{\text { References }}$}


Abraham, G., Varughese, S., Thandavan, T., Iyengar, A., Fernando, E., Naqvi, S.A., Sheriff, R., Ur-Rashid, H., Gopalakrishnan, N. and Kafle, R.K. (2016). Chronic kidney disease hotspots in developing countries in South Asia. Clinical kidney journal, 9(1), pp.135-141.

Athuraliya, N. T. C., Abeysekera, T. D. J., Amerasinghe, P. H., Kumarasiri, R., Bandara, P., Karunaratne, U., Milton, A. H. and Jones, A. L. (2011) Uncertain etiologies of proteinuricchronic kidney disease in rural Sri Lanka, Kidney international, Elsevier, 80(11), pp. 1212 1221.

Balagué, F., Mannion, A. F., Pellisé, F. and Cedraschi, C. (2012) Non-specific low back pain, The Lancet, Elsevier, 379(9814), pp. 482-491.

Bhatt, D. L., Kandzari, D. E., O’neill, W. W., D’agostino, R., Flack, J. M., Katzen, B. T., Leon, M. B., Liu, M., Mauri, L. and Negoita, M. (2014) A controlled trial of renal denervation for resistant hypertension, New England Journal of Medicine, Mass Medical Soc, 370(15), pp. 1393-1401.

Buowari, O. Y. (2013) Complications of venepuncture, Advances in Bioscience and Biotechnology, Scientific Research Publishing, 4(1), p. 126.

Centers for Disease Control and Prevention. (2017). National chronic kidney disease fact sheet, 2017. Atlanta, GA: US Department of Health and Human Services, Centers for Disease Control and Prevention.

Chehade, H., Cachat, F., Jannot, A.-S., Meyrat, B.-J., Mosig, D., Bardy, D., Parvex, P. and Girardin, E. (2013) Combined serum creatinine and cystatin C Schwartz formula predicts kidney function better than the combined CKD-EPI formula in children, American journal of nephrology, Karger Publishers, 38(4), pp. 300-306.

Davison, S. N., Koncicki, H. and Brennan, F. (2014) Pain in chronic kidney disease: a scoping review, In Seminars in dialysis, Wiley Online Library, pp. 188-204.

Davison, S.N. and Jhangri, G.S. (2010). Impact of pain and symptom burden on the health related quality of life of hemodialysis patients. Journal of pain and symptom management, 39(3), pp 477-485.

Delanghe, J. R. and Speeckaert, M. M. (2011) Creatinine determination according to Jaffewhat does it stand for?, Nephrology Dialysis Transplantation Plus, Oxford University Press, 4(2), pp. 83-86.

Florkowski, C. M. and Chew-Harris, J. S. C. (2011) Methods of estimating GFR-different equations including CKD-EPI, The Clinical Biochemist Reviews, The Australian Association of Clinical Biochemists, 32(2), p. 75.

Gillham, B. (2008) Developing a questionnaire, A\&C Black.

Gunatilake, S., Samaratunga, S. and Rubasinghe, R. (2015) Chronic Kidney Disease (CKD) in Sri Lanka-Current Research Evidence Justification: A Review, Sabaragamuwa University Journal, The Sabaragamuwa University of Sri Lanka, 13(2).

Hashizume, H., Konno, S., Takeshita, K., Fukui, M., Takahashi, K., Chiba, K., Miyamoto, M., Matsumoto, M., Kasai, Y. and Kanamori, M. (2015) Japanese orthopaedic association back pain evaluation questionnaire (JOABPEQ) as an outcome measure for patients with low back pain: reference values in healthy volunteers, Journal of Orthopaedic Science, Springer, 20(2), pp. 264-280. 
Hemmelgarn, B. R., Manns, B. J., Lloyd, A., James, M. T., Klarenbach, S., Quinn, R. R., Wiebe, N., Tonelli, M. and Network, A. K. D. (2010) Relation between kidney function, proteinuria, and adverse outcomes, Jama, American Medical Association, 303(5), pp. 423429.

Herath, C., Jayasumana, C., De Silva, P. M. C. S., De Silva, P. H. C., Siribaddana, S. and De Broe, M. E. (2018) Kidney diseases in agricultural communities: a case against heat-stress nephropathy, Kidney international reports, Elsevier, 3(2), pp. 271-280.

Hsu, H.-J., Yen, C.-H., Hsu, K.-H., Wu, I.-W., Lee, C.-C., Hung, M.-J., Sun, C.-Y., Chou, C.C., Chen, Y.-C. and Hsieh, M.-F. (2014) Factors associated with chronic musculoskeletal pain in patients with chronic kidney disease, BMC nephrology, BioMed Central, 15(1), p. 6.

Jayatilake, N., Mendis, S., Maheepala, P. and Mehta, F. R. (2013) Chronic kidney disease of uncertain aetiology: prevalence and causative factors in a developing country, $B M C$ nephrology, BioMed Central, 14(1), p. 180.

Jayasekara, J. M., Dissanayake, D. M., Adhikari, S. B. and Bandara, P. (2013) Geographical distribution of chronic kidney disease of unknown origin in North Central Region of Sri Lanka, Ceylon Med J, Citeseer, 58(1), pp. 6-10.

Jayasumana, C., Paranagama, P. A., Amarasinghe, M. D., Wijewardane, K., Dahanayake, K. S., Fonseka, S. I., Rajakaruna, K., Mahamithawa, A. M. P., Samarasinghe, U. D. and Senanayake, V. K. (2013) Possible link of chronic arsenic toxicity with chronic kidney disease of unknown etiology in Sri Lanka,.

Kafkia, T., Chamney, M., Drinkwater, A., Pegoraro, M. and Sedgewick, J. (2011) Pain in chronic kidney disease: prevalence, cause and management, Journal of renal care, Wiley Online Library, 37(2), pp. 114-122.

Keller, B. J., Martini, S., Sedor, J. R. and Kretzler, M. (2012) A systems view of genetics in chronic kidney disease, Kidney international, 81(1), pp. 14-21.

Lopez-Giacoman, S. and Madero, M. (2015) Biomarkers in chronic kidney disease, from kidney function to kidney damage, World journal of nephrology, Baishideng Publishing Group Inc, 4(1), p. 57.

Manias, E. and Williams, A. (2008) Managing pain in chronic kidney disease: patient participation in decision-making, Journal of Advanced Nursing, Wiley Online Library, 61(2), pp. 201-210.

Mills, K. T., Xu, Y., Zhang, W., Bundy, J. D., Chen, C.-S., Kelly, T. N., Chen, J. and He, J. (2015) A systematic analysis of worldwide population-based data on the global burden of chronic kidney disease in 2010, Kidney international, Elsevier, 88(5), pp. 950-957.

Noble, A., Amerasinghe, P., Manthrithilake, H. and Arasalingam, S. (2014) Review of literature on chronic kidney disease of unknown etiology (CKDu) in Sri Lanka, IWMI.

Patel, M. V, Gupta, S. N. and Patel, N. G. (2011) Effects of Ayurvedic treatment on 100 patients of chronic renal failure (other than diabetic nephropathy), $A y u$, India, Medknow Publications \& Media Pvt Ltd, 32(4), pp. 483-486.

Peake, M. and Whiting, M. (2006) Measurement of Serum Creatinine - Current Status and Future Goals, Clinical Biochemist Reviews, The Australian Association of Clinical Biochemists, 27(4), pp. 173-184.

Rana, S. V (2012) No Preanalytical Errors in Laboratory Testing: A Beneficial Aspect for Patients, Indian Journal of Clinical Biochemistry, India, Springer-Verlag, 27(4), pp. 
Rajapakse, S. and Shivanthan, M. C. (2016) Chronic kidney disease of unknown etiology in Sri Lanka, International Journal of Occupational and Environmental Health, Taylor \& Francis, pp. 1-6.

Redmon, J. H., Elledge, M. F., Womack, D. S., Wickremashinghe, R., Wanigasuriya, K. P., Peiris-John, R. J., Lunyera, J., Smith, K., Raymer, J. H. and Levine, K. E. (2014) Additional perspectives on chronic kidney disease of unknown aetiology (CKDu) in Sri Lanka-lessons learned from the WHO CKDu population prevalence study, BMC nephrology, BioMed Central, 15(1), p. 125.

Redmon, J. H., Elledge, M. F., Wanigasuriya, K. P., Wickremasinghe, R. and Levine, K. E. (2016) Deciphering chronic kidney disease of unknown etiology in Sri Lanka, In RTI Press Publication.

Rowley, J. (2014) Designing and using research questionnaires, Management Research Review, Emerald Group Publishing Limited, 37(3), pp. 308-330.

Salomons, G. S. and Wyss, M. (2007) Creatine and creatine kinase in health and disease, Springer Science \& Business Media.

Saris, W. E. and Gallhofer, I. N. (2014) Design, evaluation, and analysis of questionnaires for survey research, John Wiley \& Sons.

Saunders, W. B. (2007) KDOQI clinical practice guidelines and clinical practice recommendations for diabetes and chronic kidney disease, Citeseer.

Senanayake, S., Gunawardena, N., Palihawadana, P., Bandara, P., Haniffa, R., Karunarathna, R. and Kumara, P. (2017) Symptom burden in chronic kidney disease; a population based cross sectional study, BMC nephrology, BioMed Central, 18(1), p. 228.

Shlipak, M. G., Sarnak, M. J., Katz, R., Fried, L. F., Seliger, S. L., Newman, A. B., Siscovick, D. S. and Stehman-Breen, C. (2005) Cystatin C and the risk of death and cardiovascular events among elderly persons, New England Journal of Medicine, Mass Medical Soc, 352(20), pp. 2049-2060.

Stevens, L. A., Coresh, J., Greene, T. and Levey, A. S. (2006) Assessing kidney functionmeasured and estimated glomerular filtration rate, New England Journal of Medicine, Mass Medical Soc, 354(23), pp. 2473-2483.

Syal, K., Banerjee, D. and Srinivasan, A. (2013) Creatinine Estimation and Interference, Indian Journal of Clinical Biochemistry, India, Springer-Verlag, 28(2), pp. 210-211.

Theodora, K., Melissa, C., Anna, D., Marisa, P. and John, S. (2011) PAIN IN CHRONIC KIDNEY DISEASE: PREVALENCE, CAUSE AND MANAGEMENT, Journal of Renal Care, Wiley/Blackwell (10.1111), 37(2), pp. 114-122.

Valcke, M., Levasseur, M.-E., da Silva, A. S. and Wesseling, C. (2017) Pesticide exposures and chronic kidney disease of unknown etiology: an epidemiologic review, Environmental Health, BioMed Central, 16(1), p. 49.

Van de Watering, L. M. G. and Brand, A. (2008) Effects of Storage of Red Cells, Transfusion Medicine and Hemotherapy, Lörracherstrasse 16 a, Freiburg, Germany .

Wanigasuriya, K. (2012) Aetiological factors of Chronic Kidney Disease in the North Central Province of Sri Lanka: A review of evidence to-date, Citeseer.

Wanigasuriya, K. P., Peiris-John, R. J. and Wickremasinghe, R. (2011) Chronic kidney disease of unknown aetiology in Sri Lanka: is cadmium a likely cause?, BMC nephrology, 
BioMed Central, 12(1), p. 32.

Weaver, V. M., Fadrowski, J. J. and Jaar, B. G. (2015) Global dimensions of chronic kidney disease of unknown etiology $(\mathrm{CKDu})$ : a modern era environmental and/or occupational nephropathy?, BMC nephrology, BioMed Central, 16(1), p. 145.

WHO (2012) Quality assurance in bacteriology and immunology, WHO Regional Office for South-East Asia.

Wimalawansa, S. J. and Wimalawansa, S. A. (2014) Impact of changing agricultural practices on human health: chronic kidney disease of multi-factorial origin in Sri Lanka, Wudpecker Journal of Agricultural Research.

Wijekoon, R. M. A., Perera, N. A. M. and Herath, H. (2017) The Prevalence Of Chronic Kidney Disease Among Patients Diagnosed With Solid Malignancies At Teaching Hospital Anuradhapura And The Impact On Treatment Decisions., Journal of Cancer Research \& Therapeutics, 13.

Wijetunge, S., Ratnatunga, N. V. I., Abeysekera, T. D. J., Wazil, A. W. M. and Selvarajah, M. (2015) Endemic chronic kidney disease of unknown etiology in Sri Lanka: Correlation of pathology with clinical stages, Indian journal of nephrology, Wolters Kluwer--Medknow Publications, 25(5), p. 274.

Yin, P., Lehmann, R. and Xu, G. (2015) Effects of pre-analytical processes on blood samples used in metabolomics studies, Analytical and Bioanalytical Chemistry, Berlin/Heidelberg, Springer Berlin Heidelberg, 407(17), pp. 4879-4892. 
Appendix

\section{KIDNEY DISEASE AND QUALITY OF LIFE SHORT FORM (KDQOL - SF ${ }^{\mathrm{TM}}$ )}

Questionnaire

Name -

Occupation -
Age Male / Female

\section{YOUR HEALTH}

1. In general, you can say that your health is:

$\begin{array}{ccccc}\text { Excellent } & \text { Very good } & \text { Good } & \text { Fair } & \text { Poor } \\ 1 & 2 & 3 & 4 & 5\end{array}$

2. Compared to a year, what do you think about your health today?

Better now than last year ......................... 1

It's a bit better now than last year ........... 2

Almost the same as last year .................. 3

It's a bit worse now than last year ........... 4

Today's worse than last year .................... 5

3. The following items or items are about the activity you are likely to perform on a typical day. Is your health today limiting you to the following activity? If so, how much?

$\begin{array}{ccc}\text { Yes, Limited } & \text { Yes, Limited } & \text { No, not } \\ \text { a lot } & \text { a little } & \text { Limited }\end{array}$

a. Tired activities such as running, lifting of heavy, and involvement in heavy sports

2

b. Moderate activities, such moving a table, sweeping, bowling or walking 30 minutes

c. lifting or carrying groceries

d Climbing several flights of stairs

123

e. Climbing one flight of stairs

f. Bending, kneeling or stooping

123

g. Walking more than a mile

123

123

123

123

4. During the past four weeks, have you had the following problems in your job or any regular activity because of your health condition?

a. Cut down the amount of time you spent on your job or other activity?

$\begin{array}{cc}\text { Yes } & \text { No } \\ 1 & 2 \\ 1 & 2 \\ 1 & 2 \\ 1 & 2\end{array}$

d. Had difficulty performing work or other activities?

(for example, you need extra effort) 
5. During the past 4 weeks, to what extents have your physical health or emotional problem interfered with your normal social activities with your family, relatives, neighbors or groups?

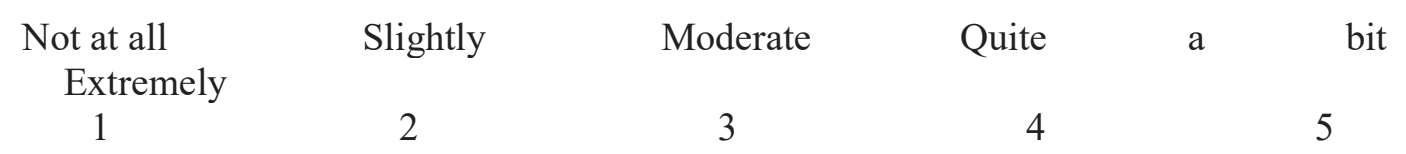

6. How much bodily pain have you had during the past 4 weeks?

None Very mild Mild Moderate Severe Very Severe

$\begin{array}{llllll}1 & 2 & 3 & 4 & 5 & 6\end{array}$

7. During the past 4 weeks, how much did you pain interfere with your normal work (including outside and indoor activities)?

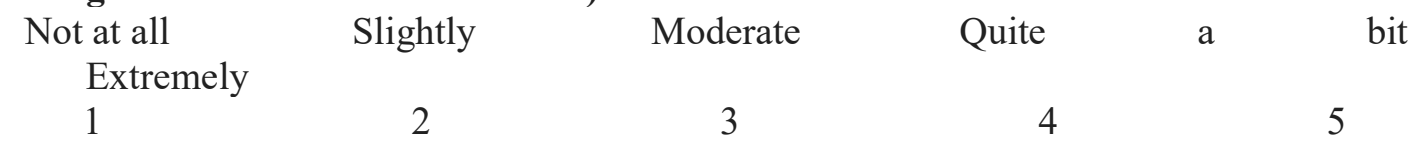

8. During the past 4 weeks, how much of the time have your physical health or emotional problems interfered with your social activities (like visiting friends, relatives, etc)?
All of the times
Most of the time
Some of the time
1
2
3
A little of the time
None of the time
4
5

9. How TRUE or FALSE is each of the following statements for you.

$\begin{array}{ccccc}\text { Definitely } & \text { Mostly } & \text { Do Not } & \text { Mostly } & \text { Definitely } \\ \text { True } & \text { True } & \text { know } & \text { False } & \text { False }\end{array}$

a. My kidney disease

Interferes too much with life

b Too much of my time is spent dealing with my kidney disease

1

2

3

4

5

c. I feel frustrated dealing with my kidney disease

d. I feel like a burden of my family

1

2

3

5 
10. During the past 4 weeks, to what extent were you bothered by each of the following?

$\begin{array}{ccccc}\begin{array}{c}\text { Not at all } \\ \text { bothered }\end{array} & \begin{array}{c}\text { Somewhat } \\ \text { bothered }\end{array} & \begin{array}{c}\text { Moderately } \\ \text { bothered }\end{array} & \begin{array}{c}\text { Very much } \\ \text { bothered }\end{array} & \begin{array}{c}\text { Extremely } \\ \text { bothered }\end{array} \\ 1 & 2 & 3 & 4 & 5 \\ 1 & 2 & 3 & 4 & 5 \\ 1 & 2 & 3 & 4 & 5\end{array}$

If you think your sleep is half-way between " very bad" and "very good", circle the number 5 . If you think your sleep is one level better than 5 , circle the number 6 . If you think your sleep is worse than one level than 5 , circle the number 4 .

11. On a scale from 0 to 10 , how would you rate your sleep overall?

Very bad Very good

\begin{tabular}{|l|l|l|l|l|l|l|l|l|l|l|l|}
0 & 1 & 2 & 3 & 4 & 5 & 6 & 7 & 8 & 9 & 10 \\
\hline & & & & & & & & & &
\end{tabular}

12. Overall, how would rate your health?

$\begin{array}{ccc}\text { Worst possible } & \text { Half-way } & \text { Best } \\ \text { (as bad or worse } & \text { worst } & \text { possible } \\ \text { than being dead) } & \text { and best } & \text { health }\end{array}$

\begin{tabular}{|l|l|ll|l|l|l|l|l|l|l|}
0 & 1 & 2 & 3 & 4 & 5 & 6 & 7 & 8 & 9 & 10 \\
\hline & & & & & & & & &
\end{tabular}

13. Do you currently take prescription medications regularly (4 or more days a week) that are prescribed by your doctor for a medical condition? Please don't count over the counter medications like antacids or aspirin.

No ............................... 1 Please skip to Question 26
Yes .........................

\section{3a. How many different prescription medications do you currently take?}

Number of Medications:

14. How many years have you been involving in agriculture?

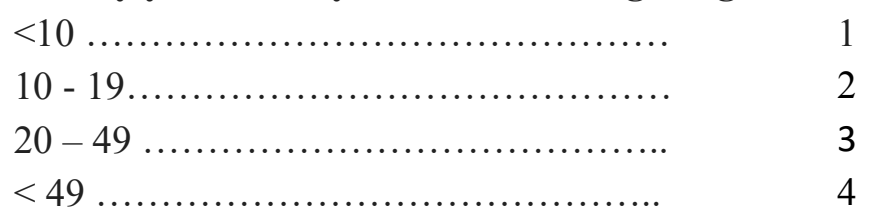




\section{What is your water resource?}

Natural springs.................... 1

Shallow wells........................ 2

Others................................. 3

\section{Which type of agriculture you have involved}

Paddy ................................... 1

Chena................................. 2

Others.................................. 3

\section{Thank you for taking part in this study.}

The questionnaire was adapted from, "Kidney disease quality of life short form", (Joshi, Mooppil and Lim, 2010)

Joshi, V. D., Mooppil, N. and Lim, J. F. Y. (2010) Validation of the Kidney Disease Quality of Life-Short Form: a cross-sectional study of a dialysis-targeted health measure in Singapore, BMC Nephrology, 11(1), p. 36.

The grading was assessed using "Pain in chronic kidney disease"

Theodora, K., Melissa, C., Anna, D., Marisa, P. and John, S. (2011) PAIN IN CHRONIC KIDNEY DISEASE: PREVALENCE, CAUSE AND MANAGEMENT, Journal of Renal Care, Wiley/Blackwell (10.1111), 37(2), pp. 114-122.

\begin{tabular}{|c|c|}
\hline Acute pain & $\begin{array}{l}\text { Pain of recent onset that is usually transient in nature, lasting from several minutes to several days or weeks. Caused by tissue damage and is } \\
\text { often associated with some degree of inflammation. }\end{array}$ \\
\hline Chronic pain & $\begin{array}{l}\text { Pain that persists beyond the usual course of an acute illness or injury (usually beyond } 3 \text { months), associated with a pattern of recurrence over } \\
\text { months or years or associated with a chronic pathological process. }\end{array}$ \\
\hline Neuropathic pain & $\begin{array}{l}\text { Pain attributed to a peripheral injury of the nervous system or the central nervous system. Destruction, infiltration and compression of the nerve } \\
\text { tissue are the causes of pain. }\end{array}$ \\
\hline Nociceptive pain & $\begin{array}{l}\text { Pain is caused by direct stimulation of peripheral nociceptors associated with tissue injury as well as inflammation but may also be excited by } \\
\text { endogenous chemical substances. }\end{array}$ \\
\hline Constant pain & Continuing pain that does not cease or let up but continues for long periods of time \\
\hline Sharp pain & Intense, severe pain \\
\hline Intermittent pain & Pain occurring occasionally or at regular or irregular intervals; periodic pain. \\
\hline Idiopathic pain & Pain of unknown cause or spontaneous origin. \\
\hline
\end{tabular}

Figure adapted from (Theodora et al., 2011). 